\title{
EFEITOS DO TREINAMENTO RESISTIDO E AERÓBIO INTERVALADO PERIODIZADOS SOBRE O ÍNDICE DE MASSA CORPORAL EM PACIENTES COM SÍNDROME METABÓLICA
}

Malu dos Santos Siqueira ${ }^{1}$, Maria Paula Ferreira de Figueiredo ${ }^{1}$, Ítalo Ribeiro Lemes ${ }^{1}$, Stephanie Nogueira Linares ${ }^{1}$, Rodolfo Augusto Travagin Miranda ${ }^{1}$, Carlos Iván Mesa Castrillón ${ }^{1}$, Larissa Rodrigues Souto ${ }^{1}$, Alcirene Policarpo de Souza ${ }^{2}$, Carlos Marcelo Pastre ${ }^{1}$, Jayme Netto Júnior ${ }^{1}$

${ }^{1}$ Universidade Estadual Paulista - UNESP, Departamento de Fisioterapia, Presidente Prudente, SP. ${ }^{2}$ Diretora no Laboratório de Análises Clínicas Marlene Spir Ltda, Presidente Prudente, SP. e-mail: malu_corao@hotmail.com

Apoio: PIBIC/CNPq.

\section{RESUMO}

O objetivo deste estudo foi analisar o efeito de 12 semanas de treinamento aeróbio intervalado (TAI) e treinamento resistido (TR), periodizados, sobre o índice de massa corporal (IMC) de pacientes com síndrome metabólica (SM). Vinte e nove pacientes com SM, idade entre 40 e 60 anos e sedentários, foram randomizados em três grupos: TAI $(n=9), T R(n=11)$ e grupo controle $(G C, n=11)$. Os treinamentos foram periodizados progressivamente, constituídos por 12 semanas e 3 sessões semanais. O grupo TAI apresentou diferença significante em relação à massa corporal quando comparado ao GC no início e final do período. O TAI e TR apresentaram valores de IMC diferentes estatisticamente do GC no início e final do período. Conclui-se que 12 semanas de TAl e TR não foram suficientes para alterar significantemente os valores de IMC em participantes com SM.

Palavras-chave: síndrome X metabólica, índice de massa corporal, exercício, estilo de vida.

\section{EFFECTS OF PERIODIZED RESISTANCE AND AEROBIC INTERVAL TRAINING ON BODY MASS INDEX IN PATIENTS WITH METABOLIC SYNDROME}

\begin{abstract}
The aim of this study was to analyze the effect of 12 weeks of aerobic interval training (AIT) and resistance training (RT), periodized, on the body mass index (BMI) of patients with metabolic syndrome (MS). Twenty nine patients with MS, aged between 40 and 60 years and sedentary, were randomized into three groups: AIT $(n=9), R T(n=11)$ and control group (CG, $n=11)$. The trainings were gradually periodized, and consisted by 12 weeks and three weekly sessions. The AIT group showed significant difference relative to the body mass when compared to the CG in pre and post training. The AIT and RT showed values of BMI different statistically to the CG in pre and post training. It was concluded that 12 weeks of AIT and TR were not enough to alter significantly the values of BMI in participants with MS.
\end{abstract}

Keywords: metabolic syndrome $\mathrm{X}$, body mass index, exercise, life style.

\section{INTRODUÇÃO}

A obesidade é um problema de saúde pública caracterizada pelo excesso de gordura corporal, decorrente do desequilíbrio energético entre o consumo e o gasto calórico ${ }^{1}$. No ano de 2014 , cerca de $13 \%$ da população mundial adulta foi classificada como obesa e $39 \%$ como acima do peso $^{2}$. No Brasil, tais números chegaram a $17,4 \%$ em adultos obesos e $51 \%$ para adultos com sobrepeso no ano de $2012^{3}$.
Dentre as diferentes formas de acúmulo de gordura no corpo, o tecido adiposo visceral caracteriza-se por apresentar resistência à insulina e aumento no número de moléculas de LDL-C e VLDL ${ }^{4}$. Sendo assim, a obesidade constitui fator de risco para diversas condições, dentre elas, a diabetes mellitus tipo 2, dislipidemia, hipertensão arterial sistêmica ${ }^{5,6}$ e síndrome metabólica (SM), sendo esta ultima fortemente associada ao excesso de peso ${ }^{7}$, tendo-o como 
principal fator de risco para seu desenvolvimento ${ }^{8-9}$.

A síndrome metabólica é um conjunto de sinais e sintomas clínicos e metabólicos, que predispõem 0 indivíduo as doenças cardiovasculares e aumenta o seu risco de morte em até 1,5 vezes. Apresenta alta prevalência na população brasileira ${ }^{10}$, e está presente em cerca de $32 \%$ dos adultos em região central brasileira ${ }^{11}$.

Estudos indicam modificações no estilo de vida como forma de prevenção e tratamento da SM, incluindo alimentação adequada e a prática de atividade física ${ }^{8,9}$. Tendo como objetivo primário a perda de peso ${ }^{8}$ e melhora do índice de massa corporal $(\mathrm{IMC})^{1,9,12}$, a atividade física regular também promove melhora no perfil lipídico, na sensibilidade a insulina e nos valores de pressão arterial ${ }^{8,9,13}$.

Em relação aos tipos de exercícios, observa-se uma grande diversidade de modalidades, sendo tanto o exercício resistido (ER) quanto o exercício aeróbio (EA) indicados. A American Heart Association ${ }^{4}$ recomenda O ER como forma de treinamento efetivo no ganho de massa magra e diminuição da massa gorda, incluindo a gordura abdominal, mediante ao aumento do gasto energético ${ }^{14}$. No entanto, o treinamento aeróbio parece ter maior efeito sobre os marcadores da $\mathrm{SM}^{15}$, atuando fortemente na diminuição da massa gorda ${ }^{16}$ por meio do tecido adiposo visceral ${ }^{8}$, no qual 0 treinamento aeróbio intervalado (TAI) tem ganho destaque, se revelando eficiente sobre os fatores da SM quando comparado ao treinamento aeróbio contínuo de intensidade moderada ${ }^{15,17}$.

Poucos estudos foram realizados comparando os efeitos do ER e do TAI sobre o IMC de indivíduos com SM, ainda apresentando resultados controversos. Neste contexto, considerando-se a obesidade como principal fator de risco da SM e o importante aumento de sua prevalência na sociedade, objetivou-se neste estudo avaliar o efeito de dois programas de treinamento periodizados sobre o IMC de participantes com SM: treinamento resistido versus treinamento aeróbio intervalado.

\section{MATERIAL E MÉTODOS}

Amostra

A amostra foi constituída por 29 voluntários sedentários de ambos os sexos com idade entre 40 e 60 anos com SM. A SM foi diagnosticada a partir da presença de 3 ou mais dos seguintes fatores: circunferência abdominal aumentada $(\geq 90 \mathrm{~cm} \mathrm{p} /$ homens e $\geq 80 \mathrm{~cm}$ para mulheres), elevação dos níveis de triglicerídeos $(\geq 150 \mathrm{mg} / \mathrm{dL})$ e glicemia de jejum $(\geq 100 \mathrm{mg} / \mathrm{dL})$, diminuição de HDL Colesterol $(<40 \mathrm{mg} / \mathrm{dL}$ p/ homens e $<50 \mathrm{mg} / \mathrm{dL} \mathrm{p} /$ mulheres) e elevação da pressão arterial $(\geq 130 \mathrm{mmHg} \text { ou } \geq 85 \mathrm{mmHg})^{18}$. Como critérios de exclusão constavam: presença de processo infeccioso, episódio de lesão músculo-tendínea ou osteoarticular nos membros superiores e inferiores e/ou coluna, doenças respiratórias, e doenças cardíacas ou neurológicas graves. $O$ presente projeto foi aprovado pelo Comitê de Ética em Pesquisa sob o número de CAAE: 31687114.8.0000.5402.

Delineamento do estudo

Os voluntários foram randomizados em 3 grupos de estudo: treinamento aeróbio intervalado (TAI, $n=9$ ), treinamento resistido ( $T R$, $\mathrm{n}=11$ ) e um grupo controle (GC, $n=9$ ). O desenvolvimento da coleta de dados se deu em uma clínica de reabilitação física em Presidente Prudente, São Paulo, para o TR, e na Faculdade de Ciências e Tecnologia, FCT-UNESP, em Presidente Prudente, em um centro de fisioterapia e reabilitação, para o TAI. As coletas das variáveis ocorreram 1 semana antes do início e na semana seguinte ao término da realização dos protocolos de treinamento.

Teste de uma repetição máxima (1RM)

O teste de 1RM foi realizado na semana anterior ao do início do treinamento para a determinação das cargas e familiarização dos voluntários com os equipamentos. Foi estabelecido o máximo de cinco tentativas para a definição desta carga máxima.

Frequência cardíaca de reserva

Para a execução do TAl, a intensidade de treinamento foi baseada em percentual da frequência cardíaca de reserva $(F C R)^{19}$. A intensidade de treinamento foi calculada pela fórmula: $[F C R=(F C m a ́ x-F C r e p) \times \%$ treinamento + FCrep], onde tem-se frequência cardíaca máxima ( $F C m a ́ x)$, frequência cardíaca de repouso (FCrep) e percentual do treinamento $(\%$ treinamento). Para o cálculo da FCmáx foi utilizada a fórmula do Karvonen descrita como FCmáx $=220$ - idade $(\text { em anos })^{20}$, e para a mensuração da FCrep foi utilizado cardiofrequencímetro da marca Polar ${ }^{\circledast}$ em repouso de 5 min na posição deitada. Para os pacientes que faziam o uso de betabloqueadores 
foi utilizada a correção da FCrep, pela fórmula: $\% \mathrm{FC}$ a corrigir $=\mathrm{Y}+95,58 / 9,74$, onde $\mathrm{Y}$ é a dose em $\mathrm{mg}$ de propranolol ou equivalente ${ }^{21}$. Para a dosagem dos medicamentos equivalentes ao propranolol utilizou-se a tabela de Kaplan $^{21}$. A porcentagem resultante desta fórmula foi então subtraída da FCmáx e posteriormente calculada a FCR.

Protocolos de treinamento

Treinamento resistido

A sessão foi divida em aquecimento (5 min em esteira ou cicloergômetro, seguido de 5 min de alongamentos gerais) e treinamento variando de 40-50 min. Foram realizados no total 8 exercícios, sendo 3 para membros inferiores (isquiotibiais, glúteos e quadríceps) e 5 para membros superiores (bíceps, tríceps, peitorais, costas e ombros). 0 treinamento teve duração de 12 semanas, sendo 3 sessões semanais, e executado de forma progressiva variando-se no início em cerca de 30 a $40 \%$ de 1 RM. Ao fim do treinamento executando cargas de até $100 \%$ do RM. A dinâmica de cargas para o TR encontra-se no Quadro 1.

Quadro 1. Dinâmica de cargas do treinamento resistido.

\begin{tabular}{|c|c|c|c|}
\hline Semanas & Sessões & $\begin{array}{l}\text { Dinâmica de Volume de Trabalho } \\
\text { Séries x Repetições por exercício }\end{array}$ & $\begin{array}{c}\text { Dinâmica de Intensidade de Esforço } \\
(1 \mathrm{RM}) \text { - Carga do exercício }\end{array}$ \\
\hline 1ㅁa & 1므, 2a e 3a & $2 \times 12$ & 30 a $40 \%$ \\
\hline $2 \underline{a}$ & $1 \underline{a}, 2 \underline{a}$ e $3 \underline{a}$ & $2 \times 16$ & 30 a $40 \%$ \\
\hline $3 \underline{a}$ & $1 \underline{a}, 2^{a}$ e $3 \underline{a}$ & $2 \times 20$ & 30 a $40 \%$ \\
\hline $4 \underline{a}$ & & Semana recuperativa & \\
\hline 5 a & $1 \underline{a}, 2^{a}$ e $3^{a}$ & $1 \times 16 / 12 / 9$ & $40 / 50 / 60 \%$ \\
\hline 6a & $1 \underline{a}, 2^{a}$ e $3 \underline{a}$ & $1 \times 12 / 9 / 6$ & $50 / 60 / 70 \%$ \\
\hline $7 \underline{a}$ & $1 \underline{a}, 2 \underline{a}$ e $3 \underline{a}$ & $1 \times 10 / 8 / 6$ & $60 / 70 / 80 \%$ \\
\hline 8 a & $1 \underline{a}, 2 \underline{a}$ e $3 \underline{a}$ & $1 \times 8 / 6 / 4$ & $70 / 80 / 90 \%$ \\
\hline 9a & & Semana recuperativa & \\
\hline $10^{a}$ & $1 \underline{a}, 2 \underline{a}$ e $3 \underline{a}$ & $1 \times 6 / 4 / 2 / 4 / 6$ & $80 / 90 / 100 / 90 / 80 \%$ \\
\hline $11 \mathrm{a}$ & $1 \underline{a}, 2^{a}$ e $3^{a}$ & $1 \times 6 / 4 / 2 / 2 / 4 / 6$ & $80 / 90 / 100 / 100 / 90 / 80 \%$ \\
\hline $12 \mathrm{a}$ & $1 \underline{a}, 2 \underline{a}$ e $3 \underline{a}$ & $1 \times 6 / 4 / 2 / 2 / 2 / 4 / 6$ & $80 / 90 / 100 / 100 / 100 / 90 / 80 \%$ \\
\hline
\end{tabular}

Treinamento aeróbio intervalado

Cada sessão foi dividida em 3 etapas: aquecimento ( $5 \mathrm{~min}$ de alongamento gerais e 5 min de caminhada na esteira com FCR inferior a $20 \%$ da FCR), treinamento e desaquecimento (5 min de caminhada na esteira com FCR inferior a $20 \%$ da FCR). A fase de treinamento foi executada de forma progressiva, com variação entre $20-90 \%$ da FCR, com pausa ativa entre os tiros em intensidade variável de $19 \%$ a $50 \%$ da RFC. No momento em que o voluntário terminou $\mathrm{O}$ esforço proposto, sua velocidade era diminuída até que o participante demorasse entre 1 a $4 \mathrm{~min}$ para atingir a FC delimitada, e desta maneira iniciou-se o próximo esforço. Na distribuição da dinâmica de cargas previstas para o TAI, o número de séries e os tempos de esforço foram fixos, e o tempo de recuperação entre as séries, tempo total e a velocidade de esforço foram variáveis e estabelecidos individualmente. Para tais variáveis houve uma tabela de controle diário para que a porcentagem da FCR estivesse sempre dentro dos parâmetros estabelecidos em cada fase de treinamento. A dinâmica de cargas de acordo com a fase de treinamento encontra-se no Quadro 2.

Avaliação antropométrica: Estatura, peso e índice de massa corpórea (IMC)

A análise da estatura foi realizada pela mensuração em posição ortostática, por meio de um estadiômetro da marca Sanny. A variável antropométrica peso foi colhida por meio da balança da marca TANITA, modelo BC - 418, Segmental Body Composition Analyzer, Iron Man/Inner Scaner, e o IMC calculado por meio da fórmula: $\mathrm{IMC}=$ massa corporal $(\mathrm{Kg}) /$ estatura $(m)^{2}$. 
Quadro 2. Dinâmica de cargas do treinamento aeróbio intervalado.

\begin{tabular}{|c|c|c|c|c|c|}
\hline $\begin{array}{l}\text { Semanas } \\
\text { (fase) }\end{array}$ & Sessões & $\begin{array}{c}\text { Série X } \\
\text { tempo de } \\
\text { esforço (min) }\end{array}$ & $\begin{array}{l}\text { Recuperação } \\
\text { entre as séries } \\
\text { (min) }\end{array}$ & $\begin{array}{c}\text { Tempo total } \\
\text { Esforço + Recuperação } \\
\text { (min) }\end{array}$ & $\begin{array}{c}\text { Intensidade da } \\
\text { RCF (\%) }\end{array}$ \\
\hline $1 \underline{a}$ & 1 a e $2^{a}$ & $5 \times 4$ & $1-4$ & $24-32$ & Treino: \\
\hline $2^{a}$ & $3 \underline{a}$ e $4 \underline{a}$ & $6 \times 4$ & $1-4$ & $29-39$ & $20-39 \%$ da \\
\hline 3 a & $5 a$ e $6 \underline{a}$ & $7 \times 4$ & $1-4$ & $34-46$ & RCF \\
\hline $4 \underline{a}$ & $7 \underline{a}, 8 \underline{a}$ e $9 \underline{a}$ & $8 \times 4$ & 1-4 & $39-53$ & Recuperação: \\
\hline Adaptação & $10^{a}, 11^{a}$ e $12^{a}$ & $9 \times 4$ & 1-4 & $44-60$ & $\begin{array}{c}19 \% \text { da RCF } \\
\text { (Leve) }\end{array}$ \\
\hline 5 & & & na recuperativa & & \\
\hline & $13^{a}$ e $14 \underline{a}$ & $4 \times 2,5$ & $1-4$ & $13-19$ & Treino: \\
\hline $\begin{array}{l}6 \underline{a} \\
7 \underline{a}\end{array}$ & $15^{a}$ e $16^{a}$ & $5 \times 2,5$ & 1-4 & $16,5-24,5$ & $40-59 \%$ da \\
\hline $\begin{array}{l}7 \underline{a} \\
8 \underline{a}\end{array}$ & $17 \mathfrak{a}$ e $18 \underline{a}$ & $6 \times 2,5$ & $1-4$ & $20-30$ & RCF \\
\hline $8 \underline{a}$ & & & $1-4$ & & Recuperação: \\
\hline Intermediária & $19 \mathrm{a}, 20^{\mathrm{a}}$ e $21^{\mathrm{a}}$ & $7 \times 2,5$ & & $23,5-41$ & $\begin{array}{l}30 \% \text { da RCF } \\
\text { (moderada) }\end{array}$ \\
\hline \multirow{2}{*}{\multicolumn{2}{|c|}{ semana recuperativa }} & & & & \\
\hline & $22 \underline{a}$ e $23 \underline{a}$ & $5 \times 1,5$ & $1-4$ & $11,5-19,5$ & $\underline{\text { Treino: }}$ \\
\hline $10^{\mathrm{a}}$ & $24 \stackrel{a}{e}$ e $25^{a}$ & $6 \times 1,5$ & $1-4$ & $14-24$ & $60-90 \% \mathrm{da}$ \\
\hline 11 a & $26 \underline{a}$ e $27 \underline{a}$ & $7 \times 1,5$ & $1-4$ & $16,5-28,5$ & RCF \\
\hline $12 \mathrm{a}$ & $28 \underline{a}$ e $29 a$ & $8 \times 1,5$ & $1-4$ & $19-33$ & Recuperação: \\
\hline Final & $30 \underline{a}$ & $9 \times 1,5$ & $1-4$ & $21,5-37,5$ & $\begin{array}{l}50 \% \text { da RCF } \\
\text { (alta) }\end{array}$ \\
\hline
\end{tabular}

\section{Análise estatística}

A distribuição quanto à normalidade dos dados foi testada por meio do teste de Kolmogorov-Smirnov. Na comparação entre os momentos de um mesmo grupo o teste adotado foi $\circ t$ de Student quando a distribuição foi normal, e não havendo distribuição normal, o teste de Mann-Whitney foi utilizado. Para comparação entre grupos, utilizou-se a técnica da análise de variância para modelos de medidas repetidas, sendo complementada com o teste de Tukey para dados paramétricos ou teste de Kruskal-Wallis, complementado pelo de teste de Dunn, para não paramétricos. Todos os dados foram analisados considerando o nível de $5 \%$ de significância.

\section{RESULTADOS}

Na Tabela 1 encontram-se os valores de média e desvio padrão dos dados antropométricos segundo grupo e momento. Nenhuma diferença foi encontrada entre os grupos em relação à idade a estatura. $O$ grupo TAl apresentou diferença em relação ao GC no momento inicial (M1) e final (M2) na variável massa corporal que se apresentou mais elevada, e ambos os grupos de treinamento apresentaram maiores valores de IMC no M1 e M2 em comparação ao GC.

Tabela 1. Média e desvio padrão das variáveis antropométricas segundo grupo e momento.

\begin{tabular}{lccccc}
\hline \multirow{2}{*}{ Variáveis } & & \multicolumn{3}{c}{ Grupos } & \multirow{2}{*}{ Valor $\mathbf{P}$} \\
\cline { 3 - 5 } & & $\begin{array}{c}\text { TAI } \mathbf{n}=\mathbf{9} \\
\text { Média } \pm \text { DP }\end{array}$ & $\begin{array}{c}\text { TR } \mathbf{n = 1 1} \\
\text { Média } \pm \text { DP }\end{array}$ & $\begin{array}{c}\text { GC } \mathbf{n = 9} \\
\text { Média } \pm \text { DP }\end{array}$ & 0,182 \\
\hline Idade & $54,4 \pm 3,2$ & $51,2 \pm 5,9$ & $49,9 \pm 5,9$ & 0,668 \\
Estatura (cm) & & $171 \pm 9,1$ & $166,8 \pm 11,7$ & $167,2 \pm 11,9$ & 0,025 \\
Massa corporal & M1 & $97,3 \pm 17^{*}$ & $90,5 \pm 12,9$ & $77,7 \pm 13,9$ & 0,058 \\
(Kg) & M2 & $96,5 \pm 17,4^{*}$ & $91 \pm 13$ & $78,6 \pm 14,9$ & 0,020 \\
IMC $\left(\mathrm{Kg} / \mathrm{m}^{2}\right)$ & $\mathrm{M} 1$ & $33,3 \pm 5^{*}$ & $32,5 \pm 4,1^{*}$ & $27,7 \pm 3,7$ & 0,034
\end{tabular}

IMC: índice de massa corporal; DP: desvio padrão; M1: momento inicial; M2: momento final. *Diferença

estatisticamente significante em relação ao grupo controle. 


\section{DISCUSSÃO}

A proposta do presente estudo foi analisar o efeito de dois programas de treinamento periodizados sobre o IMC de indivíduos sedentários com diagnóstico de SM e idade compreendida entre 40 e 60 anos. Os resultados do estudo indicaram diferenças significantes entre o TAl e o GC para a variável massa corporal no momento inicial e final, e diferenças significativas para ambos os grupos de treinamento em relação ao GC nos dois momentos de análise para a variável IMC.

\section{A American Heart Association ${ }^{4}$} recomenda o TR como importante fator integrante de um programa de atividade física para a promoção de saúde. Assim, Tseng et al. ${ }^{22}$ constatou em seu estudo que 12 semanas de TR foi capaz de diminuir significantemente a massa corpórea e IMC de jovens adultos acima do peso. Por outro lado, em estudo realizado com indivíduos com SM e obesos, Stensvold et al. ${ }^{23}$ observaram que 12 semanas de treinamento resistido não foram suficientes para diminuir significantemente os valores de massa corporal e IMC destes voluntários. O mesmo foi encontrado no estudo de Conceição et al. $^{24}$, no qual 16 semanas de TR não foram suficientes para alterar significantemente os valores de massa corporal e IMC em mulheres na pós-menopausa. Embora a duração dos protocolos de treinamento sejam similiares entre os estudos, seus resultados apresentam-se ainda controversos, o que pode estar relacionado a diferenças na interdependência volume-intensidade, sugerindo-se então padronização na dinâmica de cargas prescritas. Ainda, sabe-se que a alimentação tem influência direta sobre o ganho ou perda de massa corpórea e IMC, e que, estudos nos quais não houve modificações no consumo energético dos pacientes as mudanças foram pouco expressivas em relação a essas variáveis $^{4}$, o que pode ser encarado como resposta para o ocorrido no presente estudo, onde a ingestão energética não foi acompanhada.

Em relação ao TAl, em nosso estudo observou-se diminuição nos valores de massa corporal e IMC nos indivíduos treinados, corroborando com os resultados de Stensvold et al. $^{23}$, que indica que 12 semanas de TAl não foram suficientes para alterar significativamente os valores de tais variáveis. Entretanto, em estudo de Mora-Rodriguez et al. ${ }^{15}$, demonstra-se que 16 semanas de TAl melhoraram significantemente a massa corporal e IMC de sujeitos com SM. Desta forma, embora o EA seja amplamente sugerido para a diminuição de massa corporal e IMC, no presente estudo o TAI não modificou tais parâmetros significantemente, o que pode ser explicado devido a seu tempo de intervenção, no qual 12 semanas podem ter sido insuficientes, sugerindo que possivelmente seja necessário ao menos 16 semanas de intervenção para a obtenção de melhoras no IMC de sujeitos com SM.

Observa-se que os efeitos dos diferentes métodos do treinamento sobre o IMC de pacientes com SM são ainda inconclusivos, com presença de metodologias e resultados muito divergentes. Sugere-se então que mais estudos sejam realizados, com protocolos padronizados e randomizados, proporcionando assim melhor entendimento a respeito dos efeitos dos treinamentos resistido a aeróbio intervalado nesta população.

Como limitações deste estudo incluíramse o pequeno tamanho amostral, que pode ter causado fragilidade e vieses na interpretação dos dados, a ausência de controle ingestão calórica, que proporcionaria melhor interpretação dos resultados, e a ausência da avaliação da composição corporal dos participantes, uma vez que os parâmetros de massa magra ou gorda podem ser alterados de maneira importante sem que se reflita no IMC.

\section{CONCLUSÃO}

Conclui-se então que 12 semanas de treinamento aeróbio intervalado ou resistido periodizados não foram suficientes para diminuir significantemente o IMC de voluntários com SM.

\section{CONFLITO DE INTERESSE}

Os autores declaram não haver qualquer potencial de conflito de interesse que possa interferir na imparcialidade deste trabalho científico.

\section{REFERÊNCIAS}

1. Samson SL, Garber AJ. Metabolic syndrome. Endocrinol Metab Clin North Am. 2014;43(1):1-23. DOI: http://dx.doi.org/10.1016/j.ecl.2013.09.009 
2. Organization WH. Global status report on noncommunicable diseases. 2014.

3. Malta DC, Andrade SC, Claro RM, Bernal RTI, Monteiro CA. Trends in prevalence of overweight and obesity in adults in 26 Brazilian state capitals and the Federal District from 2006 to 2012. Rev Bras Epidemiol. 2014;17(Suppl 1):267-76. DOI: http://dx.doi.org/10.1590/1809-4503201400050021

4. Braith RW, Stewart KJ. Resistance exercise training: Its role in the prevention of cardiovascular disease. Circulation. 2006;113(22):2642-50. DOI: http://dx.doi.org/10.1161/CIRCULATIONAHA.105.5840 $\underline{60}$

5. Lavie CJ, Milani R V., Ventura HO. Obesity and Cardiovascular Disease. Risk Factor, Paradox, and Impact of Weight Loss. J Am Coll Cardiol. 2009;53(21):1925-32.

DOI:

http://dx.doi.org/10.1016/i.jacc.2008.12.068

6. Jensen $M D$, Ryan $D H$, Apovian $C M$, Ard JD, Comuzzie AG, Donato K a., et al. 2013 AHA/ACC/TOS guideline for the management of overweight and obesity in adults: A report of the American College of cardiology/American Heart Association task force on practice guidelines and the obesity society. Circulation. 2014;129(25):1-70. DOI: http://dx.doi.org/10.1161/01.cir.0000437739.71477.e $\underline{\mathrm{e}}$

7. Original A. Frequência de síndrome metabólica em crianças e adolescentes com sobrepeso e obesidade. 2007;25(3):221-6.

8. Penalva DQF. Síndrome metabólica: diagnóstico e tratamento. Rev Med (São Paulo). 2008;87(4):245-50. DOI: $\quad$ http://dx.doi.org/10.11606/issn.16799836.v87i4p245-250

9. Yamaoka K, Tango T. Effects of lifestyle modification on Metabolic Syndrome: A systematic review and meta-analysis. BMC Med. 2012;10(38):110. DOI: http://dx.doi.org/10.1186/1741-7015-10-138 10. De Carvalho Vidigal F, Bressan J, Babio N, SalasSalvadó J. Prevalence of metabolic syndrome in Brazilian adults: a systematic review. BMC Public Health. 2013;18(13):1198. DOI: http://dx.doi.org/10.1186/1471-2458-13-1198

11. Dutra E, de Carvalho K, Miyazaki É, Hamann E, Ito M. Metabolic syndrome in central Brazil: prevalence and correlates in the adult population. Diabetol Metab Syndr. 2012;4(1):20. DOI: http://dx.doi.org/10.1186/1758-5996-4-20

12. Kuhle $\mathrm{CL}$, Steffen MW, Anderson PJ, Murad MH. Effect of exercise on anthropometric measures and serum lipids in older individuals: a systematic review and meta-analysis. BMJ. 2014;4(6):e005283. DOI: http://dx.doi.org/10.1136/bmjopen-2014-005283

13. Eduardo $C$, Silva $S$, Paola A De, Nobrega ACL, Mady C, Rossi P, et al. I Diretriz Brasileira de Diagnóstico e Tratamento da Síndrome Metabólica. Arq Bras Cardiol. 2005;84:1-28.

14. Polito MD, Cyrino ES, Gerage AM, do Nascimento MA, Januário RSB. Efeito de 12 semanas de treinamento com pesos sobre a força muscular, composição corporal e triglicérides em homens sedentários. Rev Bras Med do Esporte. 2010;16(1):2932. DOI: $\quad$ http://dx.doi.org/10.1590/S1517$\underline{86922010000100005}$

15. Mora-Rodriguez R, Ortega JF, Hamouti N, Fernandez-Elias VE, Cañete Garcia-Prieto J, Guadalupe-Grau a., et al. Time-course effects of aerobic interval training and detraining in patients with metabolic syndrome. Nutr Metab Cardiovasc Dis. 2014;24(7):792-8.

DOI:

http://dx.doi.org/10.1016/j.numecd.2014.01.011

16. Donges CE, Duffield R. Effects of resistance or aerobic exercise training on total and regional body composition in sedentary overweight middle-aged adults. Appl Physiol Nutr Metab. 2012;37(3):499-509. DOI: http://dx.doi.org/10.1139/h2012-006

17. Tjonna A, Lee S, Rognmo O, Stolen T, Bye A. Aerobic interval training versus continuous moderate exercise as a treatment for the metabolic syndrome: a pilot study. Circulation. 2008;118(4):346-54. DOI: http://dx.doi.org/10.1161/CIRCULATIONAHA.108.7728 22

18. Alberti KGMM, et al. Harmonizing the metabolic syndrome: A joint interim statement of the international diabetes federation task force on epidemiology and prevention; National heart, lung, and blood institute; American heart association; World heart federation; International. Circulation. 2009;120(16):1640-5. DOI: http://dx.doi.org/10.1161/CIRCULATIONAHA.109.1926 44

19. Mezzani A, Hamm LF, Jones AM, McBride PE, Moholdt T, Stone JA, Urhausen A, Williams MA. Aerobic exercise intensity assessment and prescription in cardiac rehabilitation: a joint position statement of the European Association for Cardiovascular Prevention and Rehabilitation, the American Association of Cardiovascular and Pulmonary Rehabilitat. Eur J Prev Cardiol. 2012;32(6):327-50. DOI: http://dx.doi.org/10.1097/HCR.0b013e3182757050 20. Medicine ACMS. Diretrizes da ACMS para os testes de esforço e sua prescrição. 8.ed. ACMS; 2010.

21. I Consenso Nacional de Reabilitação Cardiovascular. Arq Bras Cardiol. 1997;69(4):267-291.

22. Tseng $M-L, H o ~ C-C$, Chen $S-C$, Huang Y-C, Lai $C-H$, Liaw Y-P. A Simple Method for Increasing Levels of High-Density Lipoprotein Cholesterol: A Pilot Study of Combination Aerobic- and Resistance-Exercise Training. Int J Sport Nutr Exerc Metab. 2013;23(3):27181.

23. Stensvold D, Slørdahl SA, Wisløff U. Effect of Exercise Training on Inflammation Status Among People with Metabolic Syndrome. Metab Syndr Relat Disord. 2012;10(4):267-72. DOI: http://dx.doi.org/10.1089/met.2011.0140

24. Conceição MS, Bonganha V, Vechin FC, Berton RPDB, Lixandrão ME, Nogueira FRD, et al. Sixteen weeks of resistance training can decrease the risk of 
metabolic syndrome in healthy postmenopausal women. Clin Interv Aging. 2013;8:1221-8. DOI: http://dx.doi.org/10.2147/CIA.S44245

Recebido para publicação em 28/08/2015

Revisado em 02/09/2015

Aceito em 10/09/2015 